\title{
A Cost-Effective Receiver-Module with Built-in Patch Antenna for Millimeter-Wave Wireless Systems
}

\author{
Yasutake Hirachi , Hiroshi Nakano and Akihito Kato* \\ Fujitsu Quantum Devices LTD., Communications Research Lab.* \\ Hachioji Daiichi-Seimei Bldg.. 11 F, 3-20-6 Myojin-cho, Hachioji-shi, Tokyo 192-0046, Japan \\ Phone : + 81-426-48-5793, Fax : +81-426-48-5872, E-mail : hirati@to.fqd.fujitsu.co.jp
}

\begin{abstract}
A cost-effective receiver-module with built-in patch antenna was completed as well as a transmitter. Both the transmitter and the receiver have the local-oscillator in each package and have no terminal for the mm-wave signal. The linear-gain and the noise-figure of the receiver without the antenna were $35 \mathrm{~dB}$ and $5.5 \mathrm{~dB}$, respectively. The measured phase-noise of the receiver were $-60 \mathrm{dBc} / \mathrm{Hz}$ at $1 \mathrm{kHz},-78 \mathrm{dBc} / \mathrm{Hz}$ at $10 \mathrm{kHz}$, and $-88.5 \mathrm{dBc} / \mathrm{Hz}$ at $100 \mathrm{kHz}$ off-carrier frequency. The average in the local-oscillation frequency variation on the temperature was 0.2 $\mathrm{ppm} / \mathrm{C}{ }^{\circ}$ The transmission system using the transmitter and the receiver with $23 \mathrm{dBi}$ horn antenna exhibited the error-free high-speed data transmission per-formance within $9 \mathrm{~m}$ in the indoor QPSK 100 Mbps wireless LAN.
\end{abstract}

\section{Introduction}

A new cost-effective RF-module which had both a few MMIC's and the small planar-antenna in one package was proposed for the drastic reduction $(1 / 100$ $\sim 1 / 1000$ ) in the cost of semiconductor devices used in the RF-block of millimeter-wave wireless systems [1]. These MMIC's are the mixer, the local-oscillator and the amplifier, and so on. The RF-module has only two kinds of terminals : one for IF-signal and the other for DC power supply. Since the RF-module has the antenna and the local-oscillator internally, it needs no terminal for the mm-wave signal, which is the most important concept in this proposed RF-module.

It was reported that the feasibility of the proposed RF-module was demonstrated by the indoor wireless data transmission experiment (8-PSK, $156 \mathrm{Mbps}$ ) using the developed transmitter module [2]. In that module, however, the local-signal was fed from an external mm-wave VCO, which meant that the concept (no terminal for the mm-wave signal) of the proposed RF-module was not realized.

In this paper, it is reported that the RF-modules (both the transmitter and the receiver) which have also the local-oscillator in each package are completed and are successfully utilized in the millimeter-wave video transmission system. The RF-characteristics of the receiver are mainly mentioned here, since those of the transmitter had been already reported [2].

\section{Completed cost-effective RF- modules}

Completed cost-effective RF-modules are shown in Fig.1. Both the transmitter Tx (upper) and the receiver Rx (lower) were developed. A window for the propagation of electromagnetic-wave is formed at the lid of the package. The package size is $50 \times 23 \mathrm{x}$ $6.5 \mathrm{~mm}^{3}$ and the window size is $11 \mathrm{x} 11 \mathrm{~mm}^{2}$. The package is made of Kovar metal and is hermetically sealed. The window is also hermetically sealed. The window material is the Borosilicate opaque glass. The size of the patch antenna used as a small planar-antenna is $1.60 \times 1.13 \mathrm{~mm}^{2}$.

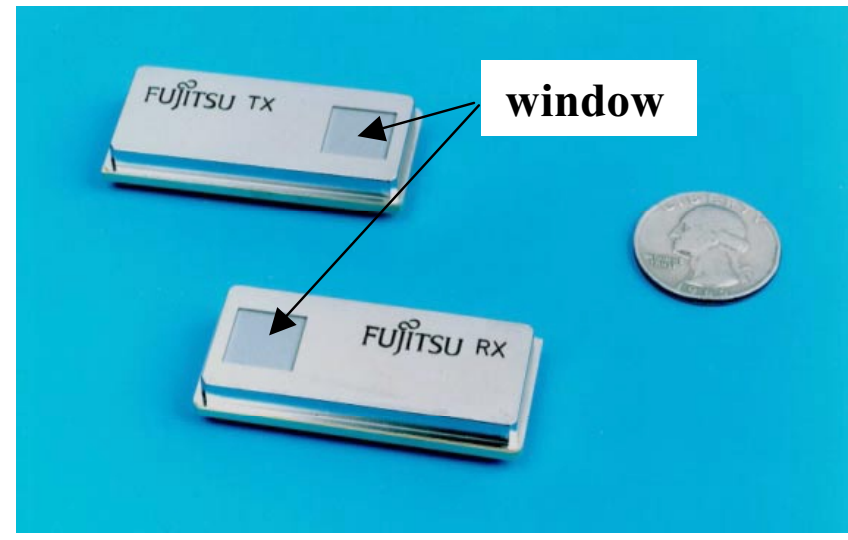

Fig.1 Completed cost-effective RF-module. The transmitter Tx (upper) and the receiver Rx (lower). The package is made of Kovar metal and is hermetically sealed. The package size is $50 \times 23 \times 6.5$ $\mathrm{mm}^{3}$ and the window size is $11 \times 11 \mathrm{~mm}^{2}$. The window material is the Borosilicate opaque glass

The block diagrams of these RF-modules are shown in Fig.2. The lineup of the transmitter (left) is almost the same as that reported so far [2], without having the local-oscillator internally. The receiver 

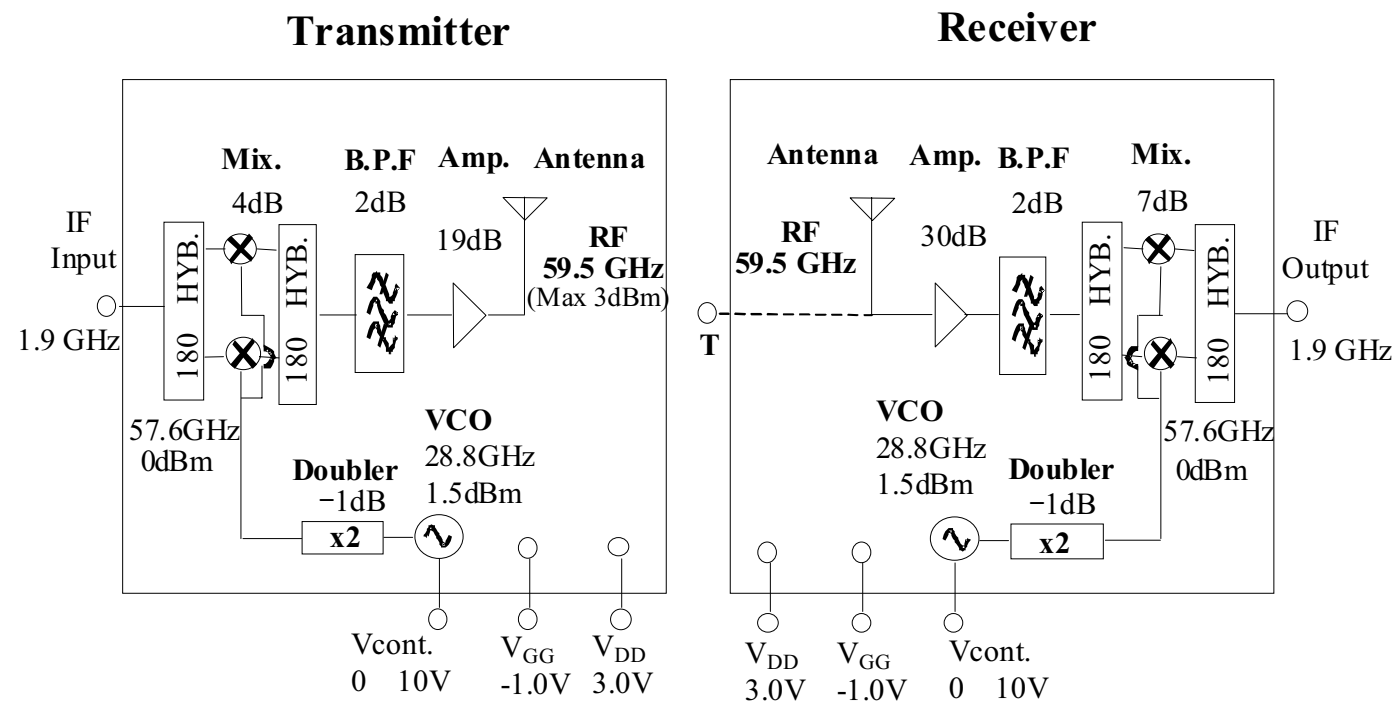

Fig.2 Block diagram of completed cost-effective RF-modules. Transmitter (left ) and Receiver (right)

(right) is composed of a patch antenna with the gain of $5.0 \mathrm{dBi}$, a MMIC amplifier with the gain of $30 \mathrm{~dB}$, a millimeter-wave band-pass filter (B.P.F) with the insertion-loss of $2.0 \mathrm{~dB}$ [3], a down-conversion MMIC balanced-mixer with the gain of $7 \mathrm{~dB}$, a Ka-band VCO with the output power of $1.5 \mathrm{dBm}[4]$, and an MMIC doubler with the amplifier and so the loss of $1 \mathrm{~dB}$. Since the down-conversion MMIC balanced-mixer has an amplifier, it can result in the conversion-gain of 7 $\mathrm{dB}$. The frequency and power of the local-signal fed to the mixer are $57.6 \mathrm{GHz}$ and $0 \mathrm{dBm}$, respectively.

\section{RF-characteristics of the receiver}

The receiver without a patch antenna but with a terminal $\mathrm{T}$ as shown in Fig. 2 was extraordinarily developed to do some experiments as shown in Fig. 3 Fig.6. In these experiments, the RF-signal of $59.5 \mathrm{GHz}$ from the synthesizer was fed to the terminal $\mathrm{T}$, and the IF-output power $\mathrm{P}_{\text {out }}$, the noise-figure $\mathrm{F}$, the phase-noise, and the temperature dependence of the local-oscillation frequency $\mathrm{f}_{\mathrm{Lo}}$ were measured at the IF-output port as shown in Fig.2. Applied voltages are $\mathrm{V}_{\mathrm{DD}}=3.0$ volts, $\mathrm{V}_{\mathrm{GG}}=-1.0$ volt, and $\mathrm{V}_{\text {cont }}=0 \sim 10$ volts, where $\mathrm{V}_{\text {cont }}$ means the control voltage of the VCO.

The measured IF-output power vs. RF received power of the receiver is shown in Fig.3. It is found that the receiver without the antenna has the linear-gain of $35 \mathrm{~dB}$ and the saturated power of -3 $\mathrm{dBm}$ at the RF-received power of $-35 \mathrm{dBm}$. The measured noise figure of the receiver is shown in Fig.4. By the band-pass filter (B.P.F), the image signal is depressed by $27 \mathrm{~dB}$, compared with the in-band signal. It can be said that the noise figure of $5.5 \square 0.7 \mathrm{~dB}$ is available

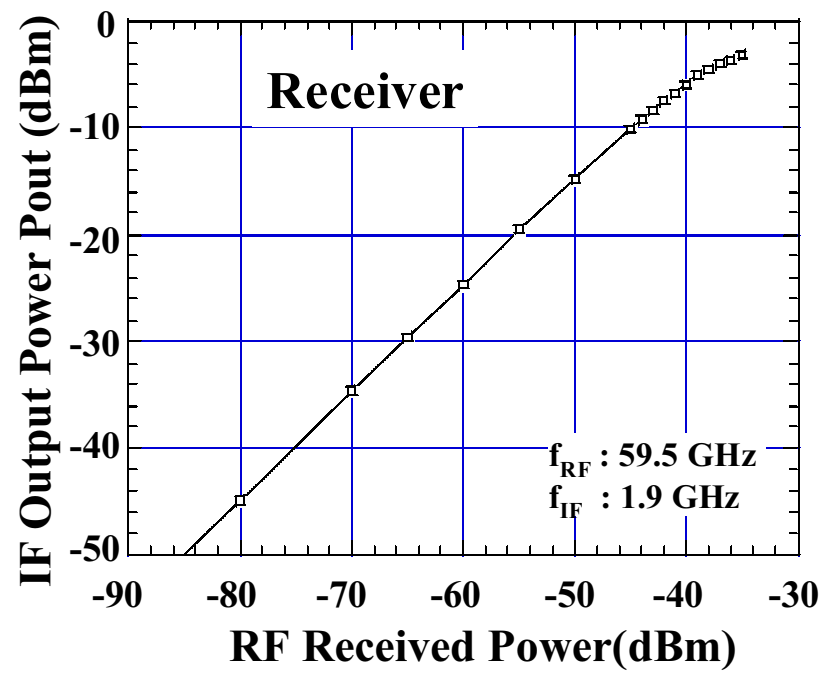

Fig. 3 Measured IF-output power vs. RF received power of the receiver.

for the practical $60 \mathrm{GHz}$ wireless system.

The measured phase-noise of the receiver is shown in Fig.5. The phase-noise was measured to be $60 \mathrm{dBc} / \mathrm{Hz}$ at $1 \mathrm{kHz},-78 \mathrm{dBc} / \mathrm{Hz}$ at $10 \mathrm{kHz}$, and -88.5 $\mathrm{dBc} / \mathrm{Hz}$ at $100 \mathrm{kHz}$ off-carrier frequency, respectively.

The temperature dependence of the local-oscillation frequency $\mathrm{f}_{\mathrm{Lo}}$ and the IF-output power $\mathrm{P}_{\text {out }}$ are shown in Fig.6. The frequency variation is expressed as the ratio $\square \mathrm{f} / \mathrm{f}_{\mathrm{Lo}}(\mathrm{ppm})\left(\mathrm{f}_{\mathrm{Lo}}=57.6\right.$ $\mathrm{GHz}$ ). The average in the frequency variation on temperature is $0.2 \mathrm{ppm} / \square$, which is also in the level of the practical use. 


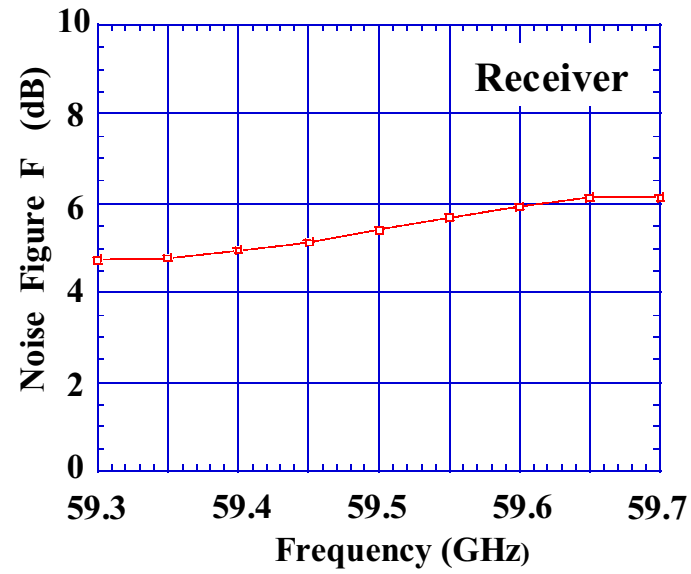

Fig. 4 Noise figure of the receiver measured at the 1.9 $\mathrm{GHz}$ IF output port. The RF-signal from the synthesizer was fed to the terminal $\mathrm{T}$ as shown in Fig. 2 (right).

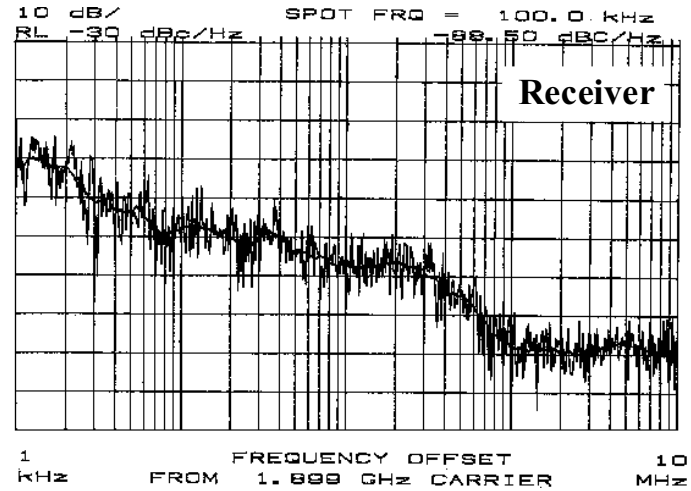

Fig.5 Measured phase-noise of the receiver. The RF-and IF-frequency were $59.5 \mathrm{GHz}$ and $1.9 \mathrm{GHz}$, respectively. The reference level indicates -30 $\mathrm{dBc} / \mathrm{Hz}$.

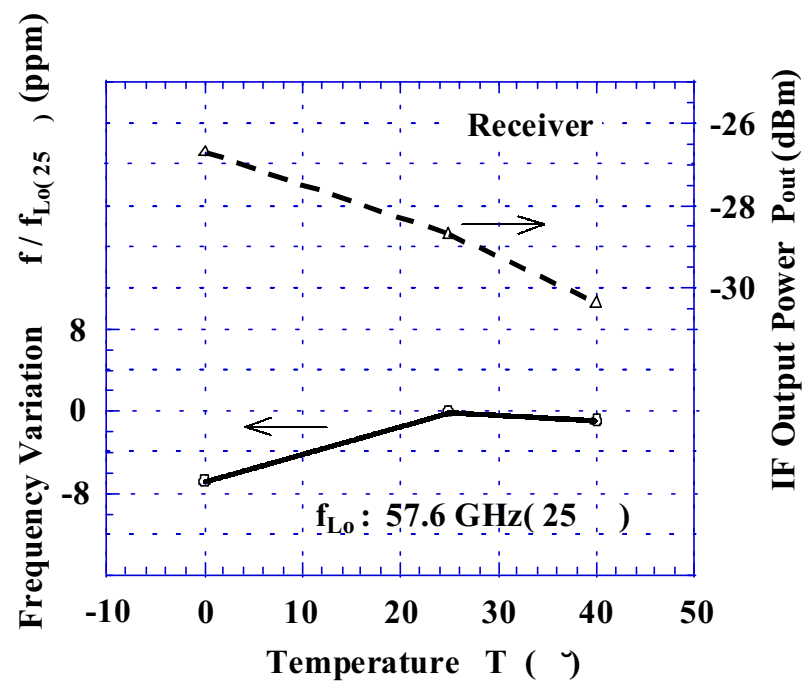

Fig. 6 Temperature dependence of the local oscillation frequency $\mathrm{f}_{\mathrm{LO}}$ and the IF output power Pout. The local oscillation frequency $\mathrm{f}_{\mathrm{LO}}$ at $\mathrm{T}=25^{\circ} \mathrm{C}$ is 57.6 $\mathrm{GHz}$.
IV. Performance evaluation of the transmitter- and receiver-modules in high-speed indoor wireless LAN system

The point-to-point wireless data transmission system was used to evaluate the performance of these RF-modules from the point of view of practical use. Figure 7 shows the setup for the high-speed indoor wireless LAN system. This wireless LAN system has the QPSK modulator and demodulator with synchronous detection. The data transmission rate is $100 \mathrm{Mbps}$.

The transmitter was mounted on the ceiling as the base-station with the elevation $\theta$ of 0,15 or 20 degree as shown in Fig.7. The receiver was located at the constant height in the experimental room. The standard horn antenna of receiver was always directed to the transmitter. The 791 bytes Ethernet packet with the speed of $100 \mathrm{Mbps}$ was transmitted continuously and the throughput and the packet loss ratio (PLR) were measured at each receiver point.

Figure 8 shows the data transmission performance at each receiver point from just under the transmitter when the elevation angle $\theta$ of transmitter was 0 degree and the azimuth angles $\phi$ were 0 or 90 degree. Almost $100 \%$ throughput of $100 \mathrm{Mbps}$ was realized within the radius of $4 \mathrm{~m}$ from just under the transmitter when the azimuth angle $\theta$ of transmitter was 0 degree.

Figure 9 shows another data transmission performance with the elevation angle $\theta$ of $0,15,20$ degree and the azimuth angle $\phi$ of 90 degree. When the elevation angle $\theta$ was 20 degree, almost $100 \%$ throughput of $100 \mathrm{Mbps}$ was realized within the radius of $9 \mathrm{~m}$ from just under the transmitter. From these evaluations, it is demonstrated that these RF-modules are suitable for the next- generation wideband wireless communication systems using $60 \mathrm{GHz}$ band.

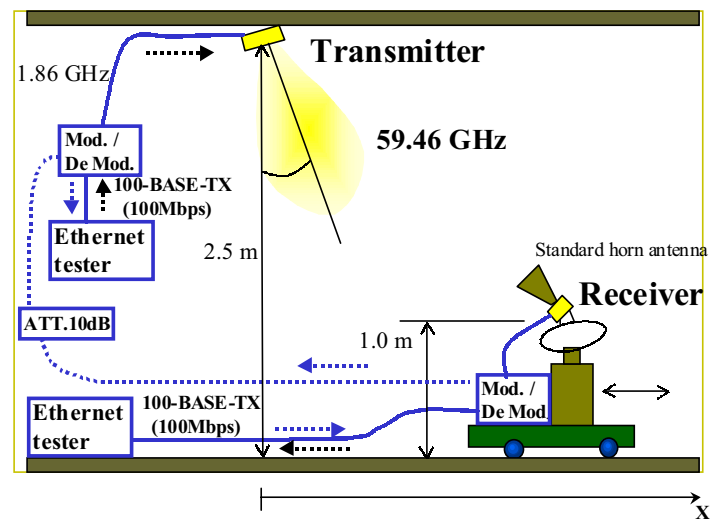

Fig.7 Setup for evaluation of indoor QPSK wireless LAN data transmission performance. Frequency : $59.46 \mathrm{GHz}$, Bit rate : $100 \mathrm{Mbps}$. The performance was evaluated at the elevation angle $\theta$ of $0,15,20$ degree. 

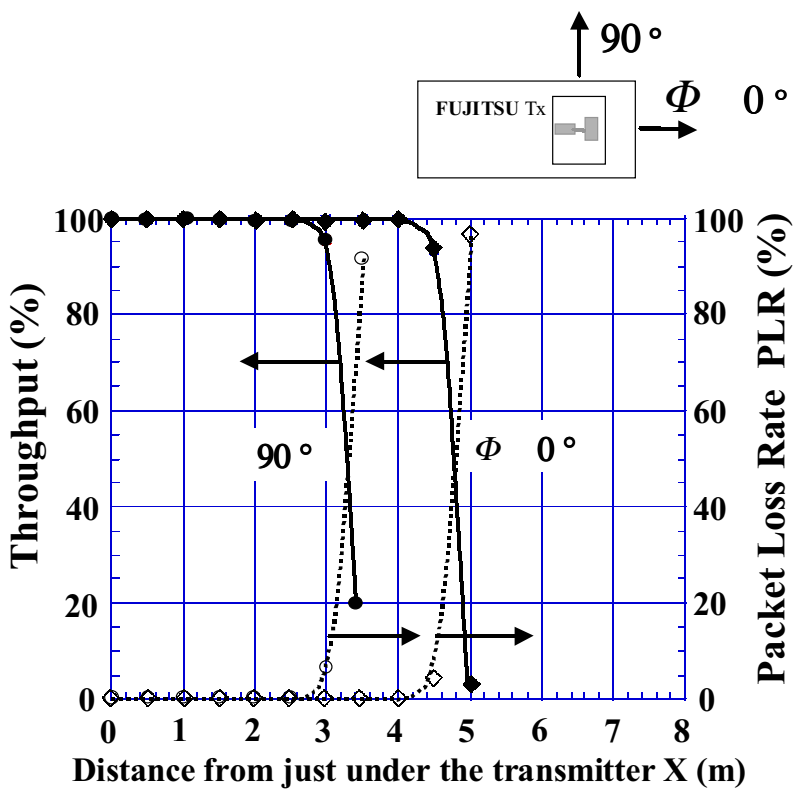

Fig. 8 Measurement results of the throughput and the packet loss ratio (PLR) versus the distance of the receiver from just under the transmitter when the elevation $\theta$ was 0 dgree and the azimuth $\phi$ was 0 or 90 degree. RF-frequency : $59.46 \mathrm{GHz}$, Bit rate : 100 Mbps.

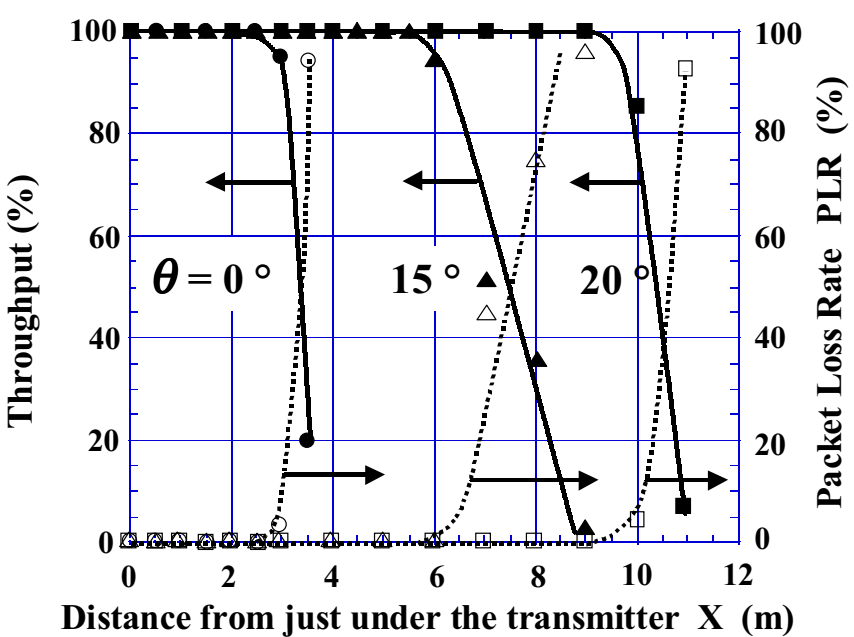

Fig.9 Measurement results of the throughput and the packet loss ratio(PLR) versus the distance of the receiver from just under the transmitter with the azimuth $\phi$ of 90 degree and the elevation $\theta=0,15,20$ degree.

\section{Conclusion}

A cost-effective receiver-module with built-in patch antenna was completed as well as a transmitter. The linear-gain and the noise-figure of the receiver without the antenna were $35 \mathrm{~dB}$ and $5.5 \pm 0.7 \mathrm{~dB}$, respectively. The measured phase-noise of the receiver were $-60 \mathrm{dBc} / \mathrm{Hz}$ at $1 \mathrm{kHz},-78 \mathrm{dBc} / \mathrm{Hz}$ at $10 \mathrm{kHz}$, and $-88.5 \mathrm{dBc} / \mathrm{Hz}$ at $100 \mathrm{kHz}$ off-carrier frequency. The average in the local-oscillation frequency variation on the temperature was $0.2 \mathrm{ppm} / \mathrm{G}$.

The transmission system using the transmitter with $5 \mathrm{dBi}$ built-in patch antenna and the receiver with $23 \mathrm{dBi}$ horn-antenna exhibited error-free high-speed data transmission performance of $100 \mathrm{Mbps}$ within 9 $\mathrm{m}$ in the indoor QPSK wireless LAN. From these results, it is demonstrated that these RF-modules are suitable for the next- generation wideband wireless communication systems using $60 \mathrm{GHz}$ band.

\section{Acknowledgement}

The authors would like to thank Dr. Y. Ishikawa and Dr. Y. Takimoto of Murata Mfg. Co., Ltd. for supplying the V-band BPF and the Ka-band VCO. They also thank Dr. M. Fujise of CRL, Mr. Y. Hirano and Mr. J. Fukaya of FQD for their support and encouragement, Mr. Y. Ohashi of Fujitsu Laboratories Ltd., Mr. J. Haryu and H. Shiratori of Fujitsu Tohoku Digital Tech., Dr. T. Tokumitsu and Mr. H. Yamawaki of FQD for their valuable discussions and experimental support.

\section{Reference}

(1) Y. Hirachi, Y. Aoki, T. Yamamoto, J. Ishibashi and A. Kato, "A cost-effective RF-module for millimeter-wave systems." 1998 Asia Pacific Microwave Conference, Yokohama Japan, pp. 53-56, TU1A-1.

(2) Y. Hirachi, H. Nakano and A. Kato, "A cost-effective RF-module with built-in patch antenna for millimeter-wave systems." $29^{\text {th }}$ Europen Microwave Conference, Conference Proc.,vol.3, pp.347-350, Munich 1999.

(3) Y. Ishikawa, T. Hiratsuka, T. Sonoda, and S. Mikami, "V band planar type dielectric resonator filter fabricated in ceramic substrate," 1997 Topical Symposium on Millimeter Waves Proc., pp.93-96.

(4) K. Sakamoto, T. Kato, S. Yamashita, and Y. Ishikawa , "A millimeter wave DR-VCO on planar type dielectric resonator with small size and low phase noise," IEICE Trans. Electron., vol. E82-C, no. 1, Jan. 1999. 